\title{
Unconventional phase separation in the model 2D spin-pseudospin system
}

\author{
K.S. Budrin ${ }^{1, \star}$, Yu.D. Panov ${ }^{1, \star \star}$, A.S. Moskvin ${ }^{1}$, and A.A. Chikov ${ }^{1}$ \\ ${ }^{1}$ Ural Federal University, Institute of Natural Sciences, 620002, 19 Mira street, Ekaterinburg, Russia
}

\begin{abstract}
The competition of charge and spin orderings is a challenging problem for strongly correlated systems, in particular, for high- $\mathrm{T}_{c}$ cuprates. We addressed a simplified static $2 \mathrm{D}$ spin-pseudospin model which takes into account both conventional spin exchange coupling and the on-site and inter-site charge correlations. Classical Monte-Carlo calculations for large square lattices show that homogeneous ground state antiferromagnetic solutions found in a mean-field approximation are unstable with respect to phase separation into the charge and spin subsystems behaving like immiscible quantum liquids. In this case, with lowering of a temperature one can observe two sequential phase transitions: first, antiferromagnetic ordering in the spin subsystem diluted by randomly distributed charges, then, the charge condensation in the charge droplets. The inhomogeneous droplet phase reduces the energy of the system and changes the diagram of the ground states. On the other hand, the ground state energy of charge-ordered state in a mean-field approximation exactly matches the numerical Monte-Carlo calculations. The doped charges in this case are distributed randomly over a system in the whole temperature range. Various thermodynamic properties of the 2D spin-pseudospin system are studied by Monte-Carlo simulation.
\end{abstract}

\section{Introduction}

The models with competing or intertwining order parameters are popular in the condensed matter theory in connection with such real systems as, for example, multiferroics or high- $\mathrm{T}_{c}$ cuprate superconductors. In cuprates, the competition of static magnetic order, bulk superconductivity and charge-density waves has attracted a lot of attention over the years, but its nature remains a challenge [1]. Earlier we suggested a simplified static 2D spin-pseudospin model $[2,3]$ which takes into account both conventional Heisenberg spin exchange coupling and the on-site and inter-site charge correlations. A detailed qualitative and quantitative analysis of the spin-charge competition within the model with the ground state (GS) and temperature phase diagrams was done in the mean field approximation (MFA). Here, in the paper, we present the results of classical Monte-Carlo (MC) calculations in a "strong" exchange limit. An interactive visualization of the actual states of the system allowed us to observe qualitatively different behavior of doped charges in the charge ordered (CO) and antiferromagnetic (AFM) phases with a clear trend to a bulk spin-charge phase separation in the AFM phase and a random distribution of the doped charges in the $\mathrm{CO}$ phase.

\section{The model}

In our model approach [4] to copper oxides such as $\mathrm{La}_{2-x} \mathrm{Sr}_{x} \mathrm{CuO}_{4}$ we assume that the on-site Hilbert space

\footnotetext{
${ }^{\star}$ e-mail: kbudrin@yandex.ru

$\star \star$ e-mail: yuri.panov@urfu.ru
}

is reduced to only three effective charge states (nominally $\left.\mathrm{Cu}^{1+; 2+3+}\right)$ of copper ions in the $\mathrm{CuO}_{2}$ planes. These charge states are associated with components of the $S=1$ pseudospin triplet with $M_{S}=-1,0,+1$, respectively. The on-site states are characterized by different hole occupation: $n_{h}=0,1,2$ for $\mathrm{Cu}^{1+; 2+; 3+}$, respectively, and different conventional spin: $s=1 / 2$ for $\mathrm{Cu}^{2+}$ and $s=0$ for $\mathrm{Cu}^{1+; 3+}$. The doped hole concentration $n$ are related to the pseudomagnetization: $n N=\sum_{i}\left\langle S_{i z}\right\rangle$. Conventional spin density for mixed valence superpositions can vary inbetween 0 and 1 in accordance with the weight of the $\mathrm{Cu}^{2+}$ in the on-site superposition.

Hereafter, in the paper we will consider only simplified spin-pseudospin Hamiltonian which takes into account the on-site and inter-site correlations, and conventional Heisenberg spin exchange coupling:

$$
\begin{aligned}
\mathcal{H}= & \Delta \sum_{i=1}^{N} S_{i z}^{2}-\mu \sum_{i=1}^{N} S_{i z}+V \sum_{\langle i j\rangle} S_{i z} S_{j z}+ \\
& +J \sum_{\langle i j\rangle} P_{0 i} \vec{s}_{i} \vec{s}_{j} P_{0 j},
\end{aligned}
$$

where the sums run over the sites of a two-dimensional square lattice, $\langle i j\rangle$ means the nearest neighbors, $S_{i z}$ and $\vec{s}_{i}$ are the on-site pseudospin and conventional spin operators, respectively. The first on-site term with $\Delta=U / 2$ relates to the on-site density-density interactions, the second term with chemical potential $\mu$ is needed to account for the charge density constraint, $n=$ const , the third term with $V>0$ describes the effects of the inter-site densitydensity interactions. The last term is the antiferromagnetic $(J>0) \mathrm{Cu}^{2+}-\mathrm{Cu}^{2+}$ Heisenberg spin exchange cou- 


\begin{tabular}{llll}
\hline GS phase & $\varepsilon=\langle\mathcal{H}\rangle / N$ & $\left\langle S_{z}\right\rangle_{j}$ & $\left\langle P_{0}\right\rangle_{j}$ \\
\hline COI & $\Delta-2 V(1-2|n|)$ & $n+(-1)^{j}(1-|n|)$ & 0 \\
COII & $|n| \Delta-2 V(1-2|n|)$ & $n+(-1)^{j}(1-|n|)$ & $(1-|n|)\left(1-(-1)^{j} \operatorname{sgn} n\right)$ \\
COIII & $(1-|n|) \Delta-2 V(1-2|n|)$ & $n+(-1)^{j}(1-|n|)$ & $|n|-(-1)^{j} n$ \\
FIM & $|n| \Delta-\frac{J}{2}(1-2|n|)$ & $n+(-1)^{j}|n|$ & $1-|n|-(-1)^{j} n$ \\
AFM & $|n| \Delta-\frac{J}{2}(1-|n|)^{2}+2 n^{2} V$ & $n$ & $1-|n|$ \\
\hline
\end{tabular}

Table 1. The mean energy $\varepsilon$, on-site charge density $\left\langle S_{z}\right\rangle_{j}$ and on-site spin density $\left\langle P_{0}\right\rangle_{j}$ of the MFA GS phases for 2D spin-pseudospin system. The index $j=0,1$ distinguishes two checkerboard sublattices.

pling, where the projection operator $P_{0 i}=1-S_{i z}^{2}$ takes into account the on-site occupation dependence. In order to study the competition of charge and spin orderings, we used in numerical calculations the Ising type spin-spin interaction with the same account of the on-site occupation dependence as in expression (1). The classical MC calculations in this case are comparable with MFA for the model (1). The most important limitation of the model is the static character of the charge and spin subsystems, since the Hamiltonian does not contain any transfer terms.

The MFA analysis [3] gives five GS solutions or phases of spin-pseudospin system. The energies and structural characteristics in terms of the on-site charge and spin densities for these phases are given in Table 1 .

In a "weak" exchange limit, at $J / 4<V$, all the GS phases (COI, COII, COIII, FIM) correspond to the various types of charge ordering. The COI is a charge-ordered phase without spin centers. In the COII and COIII phases the charge ordering is diluted by the non-interacting spins. In the FIM phase charge and spin orderings coexist. In a "strong" exchange limit, at $J / 4>V$, there are only COI and AFM phases.

\section{Numerical results}

Here we present some results of classical MC calculations in a "strong" exchange limit $(J / 4>V)$ with the heat-bath algorithm on the square lattice $256 \times 256$ under periodical boundary conditions. As an initial state, we choose the random distribution of pseudospins and spins with a fixed total $z$-component of pseudospins for a given value of $n$. We implemented high-performance parallel computing on NVIDIA graphics cards and an interactive visualization of the actual states of the system. This allows us to observe the relaxation of the system to the ground state in the process of calculation.

First, we address the on-site correlations $\Delta<0$ when they stabilize the GS COI phase. In Fig.1 the temperature dependence of the specific heat $C(T)$ and snapshots of the real states of the system at some characteristic points are shown for $\Delta=-1.5$ and $n=0.1$. The $C(T)$ dependence reveals the maximum near $T / J \approx 0.22$. The plateau at $T / J \approx 0.5$ is related to a freezing of the spin subsystem. A direct observation of the state of the system shows that

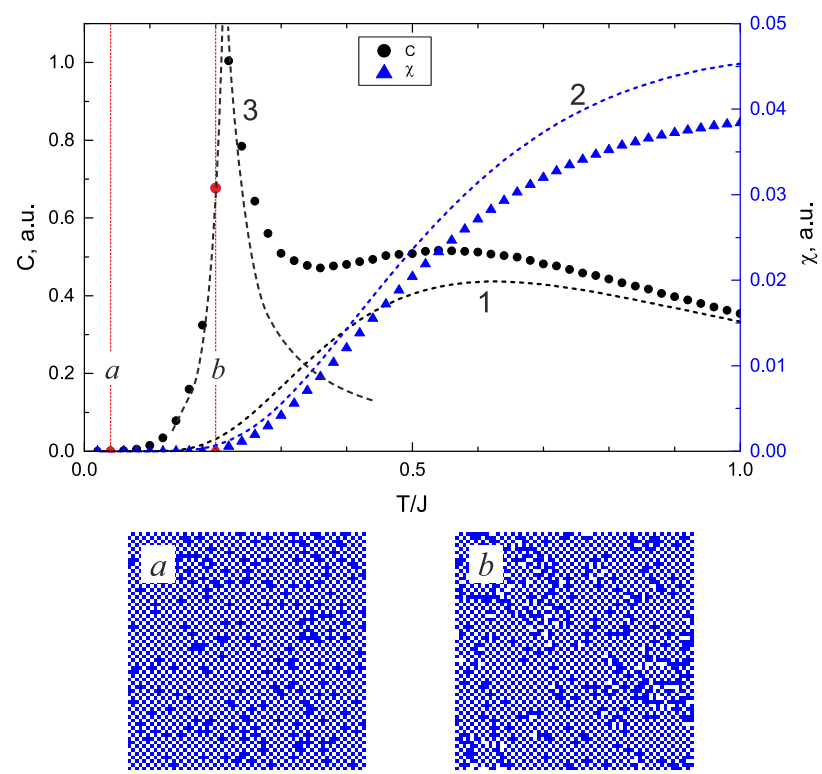

Figure 1. (colour online). The MC calculated temperature dependence of specific heat and snapshots of real states of $2 \mathrm{D}$ lattice for the CO phase at $n=0.1, \Delta=-1.5, V=0.1, J=1$. The dashed lines 1 and 2 correspond to the "free doublets" approximation $(2,3)$ for $n=0.1$ and $\Delta=-1.5$. The dashed line 3 corresponds to the Ising type dependence of specific heat for the charge subsystem given $V=0.1$. In the snapshots of real states of the lattice in the $\mathrm{CO}$ phase the blue and white colors correspond to the on-site value of $\left\langle S_{z}\right\rangle= \pm 1$, respectively. Comparison of the snapshots at temperatures $a=0.04 \mathrm{~J}$ and $b=0.2 \mathrm{~J}$ points to a weak temperature dependence of the random character of the doped charge distribution over the $\mathrm{CO}$ matrix.

the spin excitations mostly disappear at $T / J \approx 0.5$ before the ordering in the spin subsystem occurs. Qualitatively this part of the $C(T)$ dependence can be described within a rough approximation of free charge and spin doublets. In this case $V=0$ and $J=0$, so taking into account the charge density constraint we come to the expressions for the specific heat and susceptibility as follows:

$$
C=\frac{\Delta^{2} e^{\Delta / T}\left(1-n^{2}\right)^{2}}{T^{2} f\left(e^{\Delta / T}+f\right)^{2}},
$$




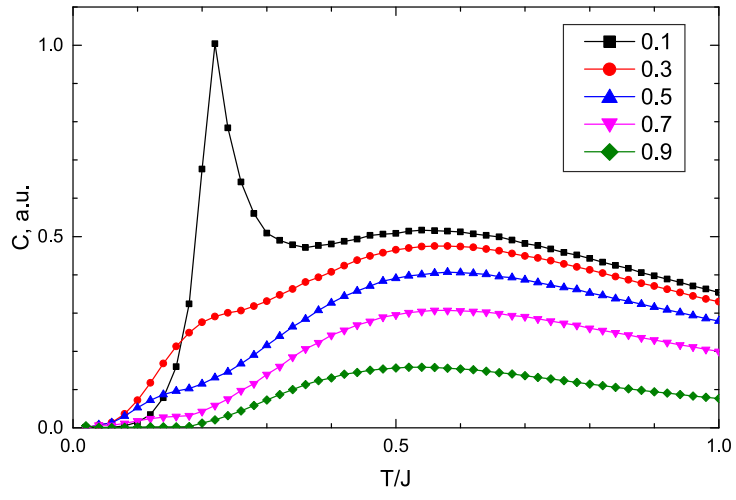

Figure 2. (colour online). The temperature dependence of the specific heat for the $\mathrm{CO}$ phase given different doping at $\Delta=-1.5$, $V=0.1, J=1$.

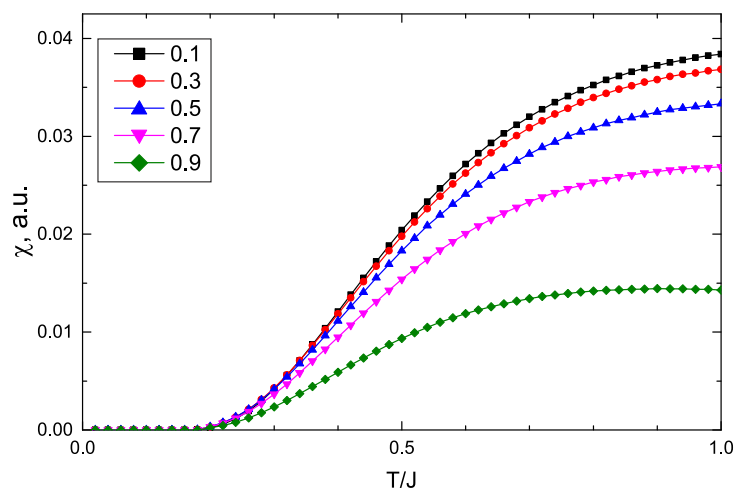

Figure 3. (colour online). The temperature dependence of the magnetic susceptibility for the $\mathrm{CO}$ phase given different doping at $\Delta=-1.5, V=0.1, J=1$.

$$
\chi=\frac{1-n^{2}}{4 T\left(1+e^{-\Delta / T} f\right)},
$$

where $f=\sqrt{1-n^{2}+n^{2} e^{2 \Delta / T}}$. The $C(T)$ and $\chi(T)$ dependencies (2) are shown in Fig. 1 for $n=0.1$ and $\Delta=-1.5$ by the dashed lines 1 and 2 , respectively.

The $C(T)$ peak at $T / J \approx 0.22$ is related to the charge ordering. The snapshots $a, b$ clearly demonstrate this feature. The temperature of the charge ordering nearly corresponds to the Ising value $T^{*}=2 \mathrm{~V} / \log (1+\sqrt{2}) \approx 2.26 \mathrm{~V}$. The distribution of doped charges over the $\mathrm{CO}$ matrix remains the random one with the temperature decrease so that the energy of the low-temperature state is exactly equal to the MFA GS energy. The concentration dependencies of the specific heat and susceptibility for the $\mathrm{CO} 1$ phase are shown in Fig.2 and Fig.3. With increasing of the charge doping the Ising type maximum of the specific heat is rapidly reduced and almost disappears above the $n \approx 0.3$. The concentration dependence of susceptibility is roughly following the expression (3).

One observes qualitatively different temperature behavior for the on-site correlations $\Delta>0$ when they stabilize the GS AFM phase. Temperature dependence of the specific heat for the AFM phase at $n=0.1$ is shown in Fig.4. The sharp maximum at $T / J \approx 0.45$ corresponds
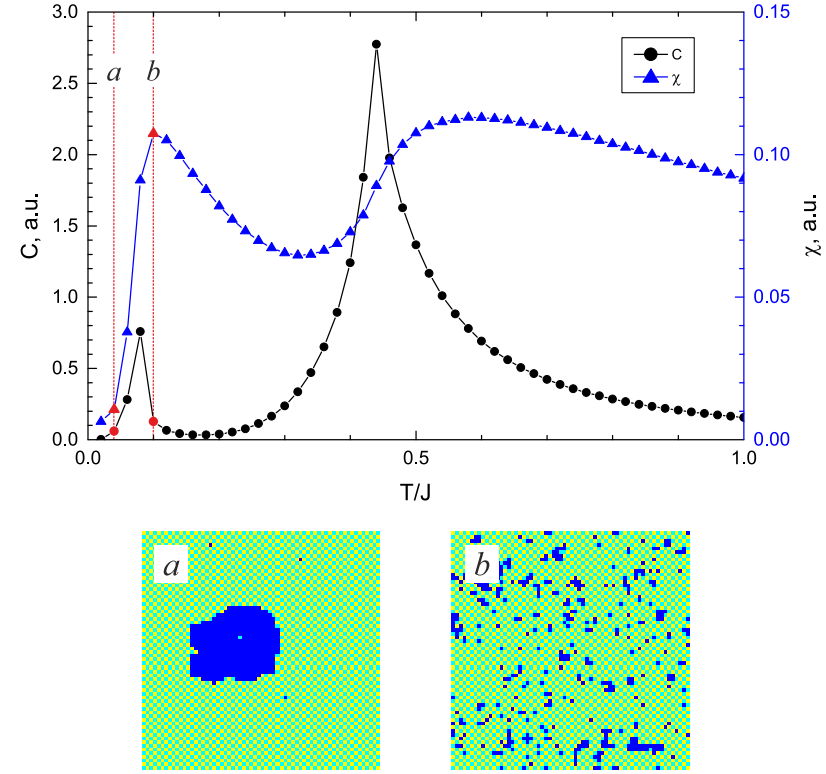

Figure 4. (colour online). The temperature dependence of the specific heat and the magnetic susceptibility for the AFM phase at $n=0.1, \Delta=0.5, V=0.1, J=1$. The Ising type hightemperature peak corresponds to the AFM ordering in spin subsystem at $T^{\star} \approx 0.45$. The low-temperature peak corresponds to the charge droplet condensation shown in the snapshots of the real states of the $2 \mathrm{D}$ lattice at the temperatures $a=0.04 \mathrm{~J}$ and $b=0.1 \mathrm{~J}$. Blue color in the snapshots points to doped charge distribution, $\left\langle S_{z}\right\rangle=1$, yellow and green colors correspond to the on-site spin values: $\left\langle s_{z}\right\rangle= \pm 1 / 2$, respectively.

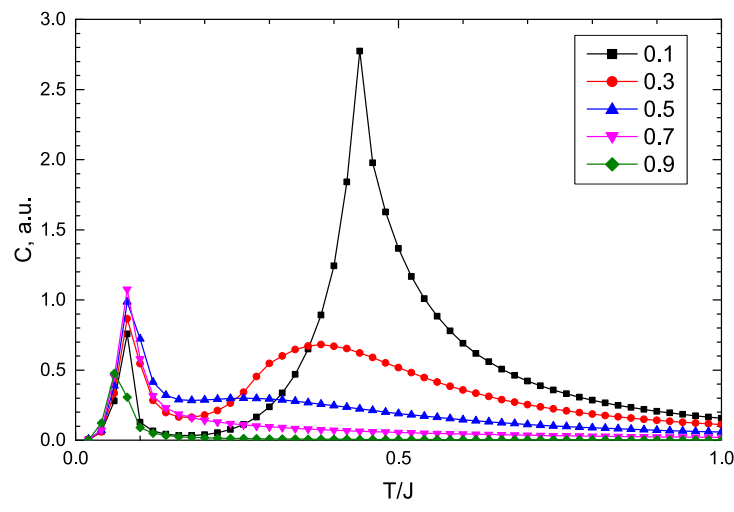

Figure 5. (colour online). The temperature dependence of the specific heat for the AFM phase given different doping at $\Delta=$ $0.5, V=0.1, J=1$.

to the AFM ordering of the spin subsystem. The temperature of the ordering nearly corresponds to the value $T^{\star} \approx 2.26\left(1-n^{*}\right) \mathrm{J} / 4$, where $n^{*} \approx 0.2$ is a total concentration of the doped and the excited charge centers. When the temperature is lowered, the specific heat demonstrates second peak at $T / J \approx 0.08$. The snapshots $a$ and $b$ show that this puzzling peculiarity is related to a condensation of doped charges in the charge droplets. The concentration dependencies of specific heat and susceptibility for the AFM phase are shown in Fig.5 and Fig.6, respectively. Phase separation in the AFM phase exists in the whole 


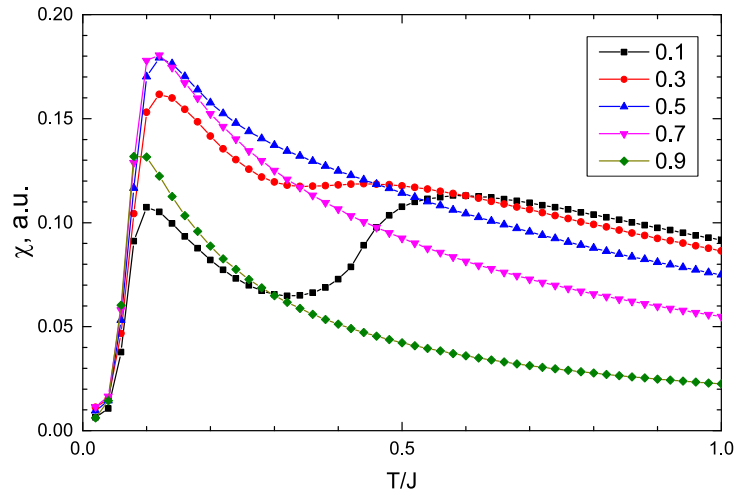

Figure 6. (colour online). The temperature dependence of the magnetic susceptibility for the AFM phase given different doping at $\Delta=0.5, V=0.1, J=1$.

range of the doped charge concentrations except for $n=0$ and $n= \pm 1$. The sharp peak on the specific heat curve at $n=0.1$ that relates to the ordering of spin subsystem rapidly flattens with the charge doping. At the same time, the second maximum does not change its position and magnitude over a wide range of concentration of the doped charge. The variation with $n$ of the susceptibility clearly indicates that the driving force of the spatial phase separation of charge and spin subsystems is related to a magnetic ordering. The calculated energy of the low temperature phase separated state is lower than the MFA GS energy of AFM phase, since the surface energy effects are omitted in MFA.

\section{Conclusions}

We have performed classical MC calculations for the 2D spin-pseudospin system with competing $\mathrm{CO}$ and spin AFM orderings in a "strong" exchange limit. The behavior of the system strongly depends on the sign and value of the on-site correlation parameter $\Delta$ either stabilizing $\mathrm{CO}$ $(\Delta<0)$ or AFM $(\Delta>0)$ phase, respectively. We show that homogeneous ground-state AFM solutions found in the MFA [1] are unstable with respect to phase separation with the charge and spin subsystems behaving like immiscible quantum liquids. The specific heat temperature dependence reveals two sequential phase transitions: first, antiferromagnetic ordering in the spin subsystem diluted by randomly distributed charges, then, the charge condensation in the charge droplets. The inhomogeneous droplet phase reduces the energy of the system and changes the diagram of the GS. Charge doping does suppress the longrange spin order, but the phase separation of doped charges and short-range spin order exists in a whole range of the charge doping. Specific heat for the system with the GS COI phase shows a feebly marked maximum due to a spin freezing at elevated temperatures with a low-temperature singular peak due to the charge ordering. The doped charges remain distributed randomly over the CO matrix up to $T=0$ since for the nearest-neighbor interaction the energies of all possible distributions of extra charges over the $\mathrm{CO}$ matrix are equal. For this reason the GS energy of the COI MFA solutions exactly matches the energy of the low-temperature MC state and the entropy of the lowtemperature state in the doped $\mathrm{CO}$ phase is higher than in the doped AFM phase.

The work is supported by Act 211 Government of the Russian Federation, agreement No 02.A03.21.0006 and by the Ministry of Education and Science, projects 2277 and 5719.

\section{References}

[1] E. Fradkin, S. A. Kivelson, J. M. Tranquada, Rev. Mod. Phys. 87, 457 (2015). DOI: 10.1103/RevModPhys.87.457

[2] Yu. D. Panov, A. S. Moskvin, A. A. Chikov, I. L. Avvakumov, J. Supercond. Nov. Magn. 29, 1077 (2016). DOI: 10.1007/s10948-016-3378-5

[3] Yu. D. Panov, A. S. Moskvin, A. A. Chikov, K. S. Budrin, J. Low Temp. Phys. 187, 646 (2017). DOI: 10.1007/s10909-017-1743-9

[4] A. S. Moskvin, Physical Review B 84, 075116 (2011). DOI: 10.1103/PhysRevB.84.075116

[5] A. S. Moskvin, JETP 148, 549 (2015). DOI: 10.1134/S1063776115090095 